\title{
A New Worker Policy for Self-Balancing Production Line with Stations
}

\author{
Daisuke Hirotani ${ }^{\dagger}$ \\ Faculty of Engineering, Hiroshima University \\ 1-4-1, Kagamiyama, Higashi-Hiroshima, 739-8527, Japan \\ E-mail: dhiro@hiroshima-u.ac.jp \\ Katsumi Morikawa \\ Faculty of Engineering, Hiroshima University \\ 1-4-1, Kagamiyama, Higashi-Hiroshima, 739-8527, Japan \\ E-mail: mkatsumi@hiroshima-u.ac.jp \\ Katsuhiko Takahashi \\ Faculty of Engineering, Hiroshima University \\ 1-4-1, Kagamiyama, Higashi-Hiroshima, 739-8527, Japan \\ E-mail: takahasi@hiroshima-u.ac.jp
}

Received, February 25, 2011; Accepted, June 4, 2011

\begin{abstract}
In traditional production lines, such as assembly lines, each worker is usually assigned to a particular fixed work, and decreasing the task to master the assigned work is valuated. However, when an imbalance exists between workers' speeds, if a worker delays the overall work in the production line, the production rate of the particular line will also decrease. To avoid this problem, the "Self-Balancing Production Line" was introduced. In this type of production line, each worker is assigned work dynamically, and when specific conditions are satisfied, production remains balanced. Characteristics of these lines that can be preempted at any place have already been analyzed by some researchers. A previous paper examined the situation in which only a single worker can process one machine and cannot preempt processing, and the improved policy of an ordinary selfbalancing production line, which specifies which stations workers can process and how workers can behave.This policy achieveda high production rate with only four stations and two workers (Buzacott, 2002). In that paper, worker processing stations and the behavior of a specific worker were limited, andthe paper focused only on specific stations and workers. Therefore, it is not applicable to any worker sequence. In this paper, we focus on other ways to decrease cycle time. In this kind of line, a worker processes at his or her speed. Therefore, if a worker is assigned stations according to his or her speed, the line can decrease cycle time. To do so, we relax the assumptions of this type of line and set a new condition. Under these conditions, we compare our results to the results of previous papers.
\end{abstract}

Keywords: Self-Balancing, Production Line, Station

\section{INTRODUCTION}

In the traditional assembly line, each worker is usually assigned to fixed work, and each worker iterates his or her assigned work continuously to achieve assembly line balancing. Balancing the work assigned to workers has been studied for the traditional line (for example, Scholl (1995)). When an imbalance in the speed of workers exists, the slowest worker will delay the overall work. As a result, the production rate of the production line will also decrease. To solve this problem, the "Self-Balancing Production Line" was introduced. The utilization of the mentioned method has been reported in at least two commercial environments: apparel manufacturing and distribution warehousing (Bartholdi and Bunimovich, 1999). In this type of production line, each worker is assigned to work dynamically, and when the last worker completes an item, he or she walks back

$\uparrow$ : Corresponding Author 
and takes over the next item from his or her predecessor. The predecessor then walks back, takes over the next item from his or her predecessor, and so on, until the first worker walks back and starts a new item. Because faster workers are assigned more work in processing an item and slower workers are assigned less, a balance can be maintained. For lines with a constant working speed, it has been found that the maximum production rate can be achieved if the workers are sequenced from slowest to fastest (Bartholdi and Eisenstein, 1996). Other conditions with three workers have been simulated numerically (Bartholdi and Bunimovich, 1999), and the performance of a production line with $n$ workers has been analyzed mathematically (Hirotani et al., 2006).

The above-mentioned papers are considered to be similar in that a worker can take over processing an item anywhere. Some papers have been published on this line with stations included. McClain et al. (2000) considered stations both with and without preemption by simulation. In their paper, six stations and three workers are assumed, and they derive the optimal zone size and control rules based on WIP (Work-In-Process) and the workers' sequence. If preemption is allowed and all workers can process anywhere, the authors claimed that the line they studied is similar to the self-balancing production line, which is the same as the line in the previous papers (ex. Bartholdi and Eisenstein, 1996).

Also, Buzacott (2002) considered stations both with and without preemption by using dynamic programming. He assumed four stations and two workers and derived an optimal policy that modifies the rule of previous selfbalancing production lines. He claimed that the modified rule can achieve higher efficiency than that of the previous self-balancing production line. Moreover, Hirotani et al. (2008) extend the above paper to any number of workers and stations. They claimed that if the number of either workers or stations is large, the modified proposed rule is better than that of the aforementioned papers.

Hirotani et al. (2009) considered the case in which worker speed is deterministic when there are some stations. They analyzed conditions for achieving the maximum production rate and based on the analysis, they proposed a policy that increased the production rate.

Armbruster and Gel (2006) considered a model in which each worker's speed can be varied according to the work both with and without passing workers. They claimed that their simple rule, which switched the sequence of the workers, is a good way to balance the line.

Nakade and Ohno (2003) proposed two kinds of allocations in U-shaped production lines: separate and carousel. Separate allocation is an allocation in which each worker processes at some assigned machine. On the other hand, Carousel allocation is an allocation in which every worker processes at all machines sequentially, like a carousel.

In this paper, based on the previous papers of Armbruster and Gel (2006) and Nakade and Ohno (2003), we propose a new worker policy to decrease cycle times. To do so, we relax the assumption regarding how this line allows passing and set a new condition. Under this situation, we compare our results to the results of previous papers.

This paper is organized as follows: In section 2, assumptions and characteristics of this self-balancing production line are explained. In section 3, we propose a new worker policy to decrease cycle time. In section 4, we show numerical examples to compare with policies of precious papers. Finally, our conclusion and other remarks are given in section 5 .

\section{THE PRODUCTION LINE}

In this section, assumptions and characteristics of the self-balancing production line used in this paper are described.

\subsection{Assumptions}

In this paper, the production line with the following assumptions is considered.

1. Each worker processes only one identical item sequentially.

2. Workers are sequenced from one to $n$ on the production line, and each worker never passes over the upstream and downstream workers.

3. A worker processes his or her work while he or she moves along the line, and worker $i$ processes at a constant speed in the production line. In this paper, we define $p_{i}$ as the processing time for worker $i$ at each station. However, the workload for each station is the same. Therefore, the processing time for each station only varies according to the worker speed.

4. When the last worker finishes processing an item, worker $n$ walks back to worker $n-1$ and takes over the next item from worker $n$-1. Then, worker $n$-1 walks back to worker $n-2$ and takes over the next item from worker $n$-2. Similarly, all workers walk back to the worker who precedes them and takes over the next item from the preceding worker, and worker 1 introduces a new item into the system. The time required to walk back and take over is ignored. If the preceding worker is still processing when he or she starts to walk back, he or she must wait until the preceding worker finishes processing at that station.

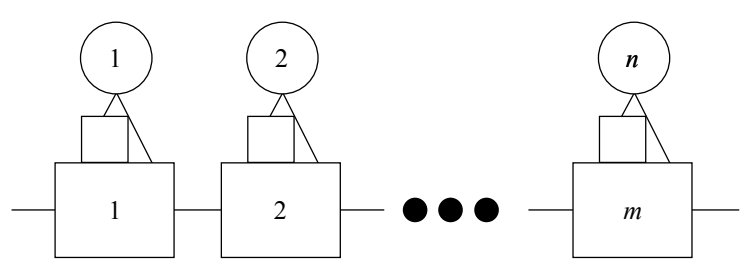

Figure 1. Production line with $m$ stations and $n$ workers. 
5. There are $m(>n)$ stations in the production line (see Figure 1). Preemption, in which a worker can take over an item from his or her predecessor while processing, is not allowed. The time required to walk between stations is ignored.

\subsection{Self-Balancing and Convergence}

It has been shown that the production line can maintain balance when workers are sequenced from slowest to fastest and the working speed is constant for all workers (Bartholdi and Eisenstein, 1996). Subsequently, the position of workers will converge to a unique fixed point, defined as $x_{i}{ }^{*}$, for worker $i$ as follows:

$$
x_{i}^{*}=\frac{\sum_{k=1}^{i-1} v_{k}}{\sum_{k=1}^{n} v_{k}}
$$

where $v_{i}$ is the speed of worker $i$. Under this condition, the production rate can be calculated as the sum of each worker's speed. Hirotani et al. (2006) found the condition for convergence that the line can balance for $n$ workers as follows:

$$
\frac{\sum_{k=1}^{i-1}(-1)^{i+k-1} v_{k}}{v_{n}}<1
$$

This condition includes the slowest-to-fastest sequence shown by Bartholdi and Eisenstein (1996).

\subsection{Imbalance}

When workers are not sequenced in such a way that the line can maintain balance, a slower worker prevents the preceding faster worker from continuing processing. This condition is called blocking. After blocking occurs, the faster worker moves at the same speed as the slower worker until the last worker finishes his or her item. Under this condition, the position of workers will not converge to a fixed point, and thus, the production rate decreases. Two kinds of blocking exist. One is blocking caused by the worker's initial position, and the other is bloc king caused by the worker's speed. In our model, additional blocking also occurs when the worker walks back, unless the predecessor has finished processing at any station.

\section{NEW WORKER POLICY}

In this section, we propose a new worker policy to decrease cycle time based on the previous papers of Armbruster and Gel (2006) and Nakade and Ohno (2003). To do so, we relax the as sumptions in this kind of line and set a new condition. The new worker policy arises based on the idea that if each worker processes according to the worker's speed, the line can be balanced. For example, if the number of stations is five and workers' processing times are $p_{1}=2, p_{2}=3$, respectively, worker 1 processes at three stations and worker 2 processes at two stations. We consider two measures: (1) allowing passing and (2) walking back.

\subsection{Allowing passing}

This measure is whether passing occurs or not. This policy is based on the previous paper of Armbruster and Gel (2006). They assumed that a worker can change his or her speed according to the work. Under this condition, allowing passing is more effective. If a downstream worker has finished an item and the processing time of the downstream worker is larger, the worker can pass the downstream worker and process an item that has been processed by the downstream worker. The downstream worker processes an item that has been processed by the other worker. In the traditional rule of selfbalancing production lines, workers cannot pass. If the sequence is not ideal for achieving a high production rate, the production rate can increase to change the sequence. We show an example for two workers and four stations with $p_{1}=1$ and $p_{2}=2$ in Figure 2. In Figure 2, a number in the box represents a worker who processes at each station. At time 2, workers 1 and 2 finish processing at stations 1 and 2, respectively, and worker 1 is faster than worker 2. Therefore, worker 1 passes worker 2 and processes an item processed by worker 2 at station 3 , while worker 2 processes an item processed by worker 1 at station 2 .

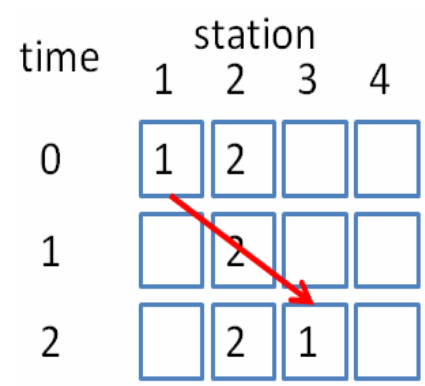

Figure 2. An example of allowing passing with $p_{1}=1$ and $p_{2}=2$.

\subsection{Walking back}

This measure is one in which the last worker walks back. In the traditional rule for a self-balancing production line, the last worker walks back to worker $n-1$. In this paper, as an alternative, the last worker walks back to the first station, such as the carousel-type allocation proposed by Nakade and Ohno (2003). We show an example for two workers and four stations with $p_{1}=2$ and $p_{2}=1$ in Figure 3. Figure 3 is similar to Figure 2. At time 3 , worker 2 finishes processing at station 4 . Therefore, worker 2 walks back to the first station. 


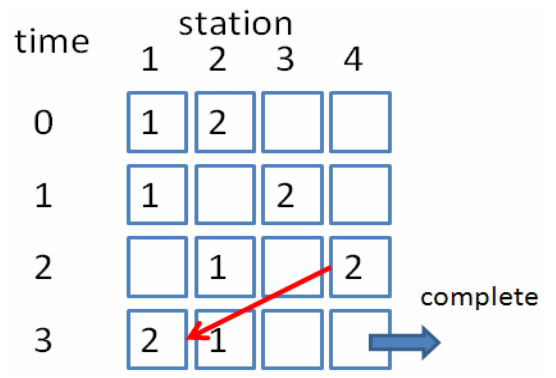

Figure 3. An example of walking back to the first station with $p_{1}=2$ and $p_{2}=1$.

\section{NUMERICAL EXAMPLE}

In this section, we show numerical examples for comparison with the traditional rule for a self-balancing production line. In our analysis, two workers $(n=2)$ and four stations $(m=4)$ are assumed. The performance measure is average cycle time.

\subsection{Effect of Allowing Passing}

We first analyze the effect of allowing passing. The condition for allowing passing is a relaxed one. Therefore, we as sumeth at cycle time decreases compared to the traditional self-balancing production line. Results are shown in Table 1. In Table 1, the cycle time of all passing cases is not better than that of no passing (traditional self-balancing production line) case. This is the opposite of what we proposed. In the previous paper by Armbruster and Gel (2006), allowing passing improves the result for achieving the maximum production rate. We consider why these results can be derived.

Table 1. Results for passing and no passing.

\begin{tabular}{|c|c|c|c|}
\hline \multirow{2}{*}{$p_{1}$} & \multirow{2}{*}{$p_{2}$} & \multicolumn{2}{|c|}{ Average cycle time } \\
\cline { 3 - 4 } & & Passing & No passing(traditional) \\
\hline \hline 1 & 2 & 3 & 3 \\
\hline 2 & 1 & 3 & 3 \\
\hline 1 & 3 & 3 & 3 \\
\hline 2 & 3 & 6 & 5 \\
\hline
\end{tabular}

\subsubsection{Case of Slowest-to-Fastest Worker Sequence}

In this case, passing never occurs. No worker is faster than other downstream workers. Therefore, the average cycle time is the same as in the case of no passing.

\subsubsection{Case of Fastest-to-Slowest Worker Sequence}

In this case, passing occurs only once. Once the worker sequence is changed, the worker sequence is slowest to fastest. Nevertheless, the case of passing is a bad result when $p_{1}=2$ and $p_{2}=3$. We show this in our figure. The variation of workers' processing stations ac-cording to the time is shown in Figure 4. In the left side of Figure 4 (passing case), worker 1 processes at stations 1 and 2 and worker 2 processes at stations 3 and 4 . This assignment is the same as fixed assignment. Therefore, worker 2 cannot take over an item from worker 1 immediately worker 2 walks back. This means that even if passing is allowed, in order to prevent blocking by the downstream worker, another blocking by an upstream worker occurs. That is why the cycle time of passing case is not better than that of the no passing case.

\subsubsection{Case that Number of Stations is Multiple of the Sum of Processing Time of All Workers}

This is a special case. For the example of $p_{1}=1$ and $p_{2}=3$, shown in Table 1 , the sum of processing times is $1+3=4$. This is the same as the number of stations (4). In this case, each worker can process an item according to the ratio of processing time. For the above parameters, worker 1 processes for three stations and worker 2 processes for only one station. Of course, this special case has no relationship with passing.
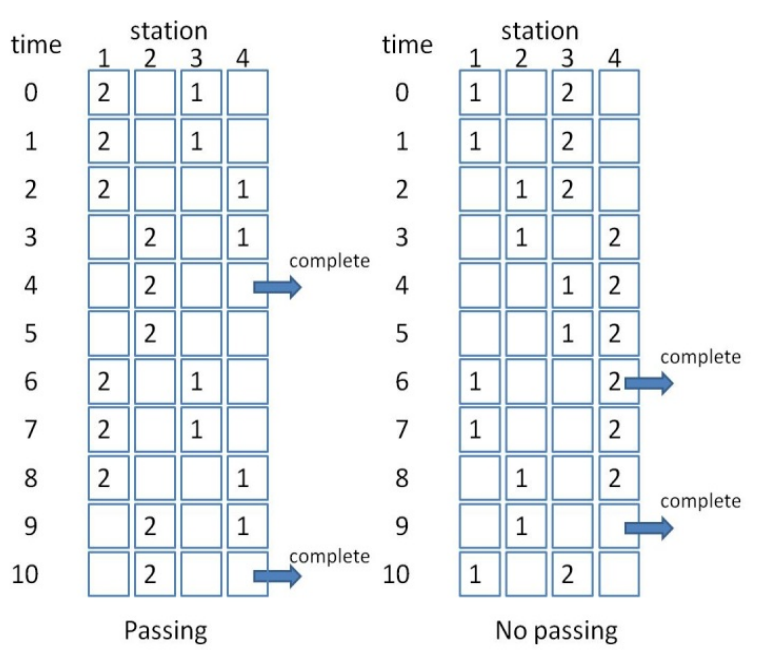

Figure 4. Worker's processing station for passing and no passing case for $p_{1}=2$ and $p_{2}=3$.

\subsection{Effect of Walking Back}

Next, we analyze the effect of walking back. Results are shown in Table 2. In Table 2, the cycle time of almost walking back to the first station case is not better than that of the worker $n-1$ case. We consider how these results can be derived.

\subsubsection{Case of Slowest-to-Fastest Worker Sequence}

In this case, slowest-to-fastest worker sequence changes to a fastest-to-slowest worker sequence. There- 
fore, blocking finally occurs. We show this with a figure for $p_{1}=2$ and $p_{2}=1$. The variation of workers' processing stations according to the time is shown in Figure 5. In Figure 5 at time 3, worker 2 has finished an item and walks back to the station 1 . At time 5 , worker 2 is blocked by worker 1 because worker 1 has not finished processing at station 3 . That is to say, another blocking occurs. That is why the cycle time of almost walking back to the first station case is not better than that of walking back to worker $n-1$ (traditional) rule. However, for $p_{1}=$ 3 and $p_{2}=2$, the cycle time of walking back to the first station case is better than that of traditional method. This is because under the traditional rule, worker 1 only processes at stations 1 and 2 and worker 2 only processes at stations 3 and 4 . This assignment is the same as the fixed assignment. That is why in this case the cycle time is better than that of the traditional rule.

Table 2. Results for the effect of walking back.

\begin{tabular}{|c|c|c|c|}
\hline \multirow{2}{*}{$p_{1}$} & $p_{2}$ & $\begin{array}{c}|c| \\
\text { Walks back to the } \\
\text { first station }\end{array}$ & $\begin{array}{c}\text { Walks back to the } \\
\text { Worker } n-1 \\
\text { (traditional) }\end{array}$ \\
\hline \hline 1 & 2 & 3.5 & 3 \\
\hline 2 & 1 & 3.5 & 3 \\
\hline 2 & 3 & 5.5 & 5 \\
\hline 3 & 2 & 5.5 & 6 \\
\hline
\end{tabular}

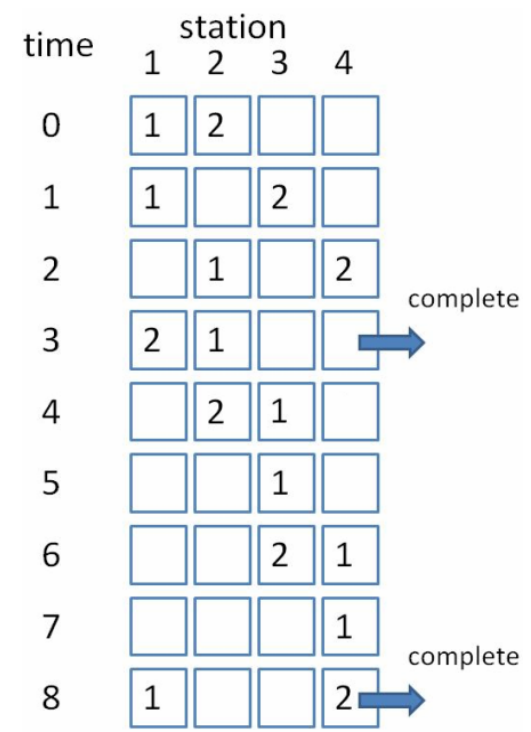

Figure 5. Worker's processing stations for the walking back to the first station case for $p_{1}=2$ and $p_{2}=1$.

\subsubsection{Case of Fastest-to-Slowest Worker Sequence}

In this case, blocking by the downstream worker occurs frequently. Therefore, faster workers have to process at the slower worker's speed. Therefore, the cycle time increases compared to the traditional rule.

\subsection{Integrating Two Policies}

In the previous sections, we show that the two policies are not better than the traditional one. This is because only one side (preventing blocking by either downstream or upstream) was considered. Next, we integrate two policies, and call this the proposed policy. Results are shown in Table 3.

Table 3. Results of comparing proposed and traditional policies.

\begin{tabular}{|c|c|c|c|}
\hline \multirow{2}{*}{$p_{1}$} & \multirow{2}{*}{$p_{2}$} & \multicolumn{2}{|c|}{ Average cycle time } \\
\cline { 3 - 4 } & & Proposed & Traditional \\
\hline \hline 1 & 2 & 2.67 & 3 \\
\hline 2 & 1 & 2.67 & 3 \\
\hline 2 & 3 & 5 & 5 \\
\hline 3 & 2 & 5 & 6 \\
\hline
\end{tabular}

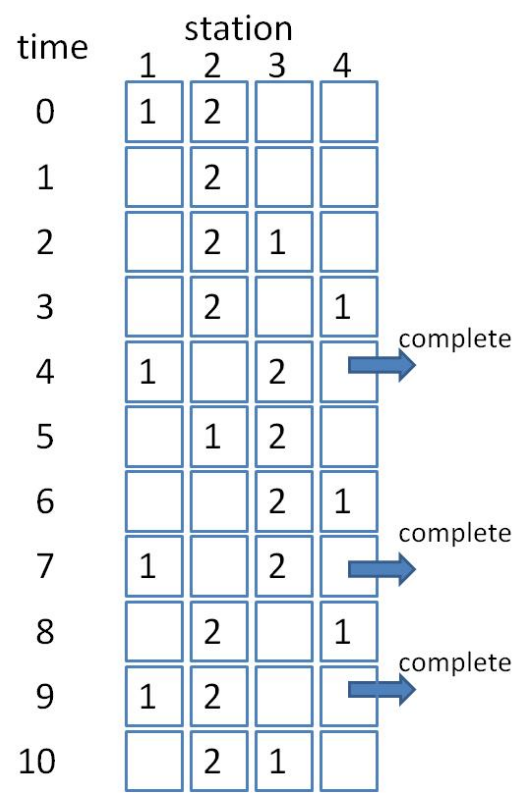

Figure 6. Workers' processing stations under the proposed policy for $p_{1}=1$ and $p_{2}=2$.

The cycle time of the proposed policy is not worse than that of the traditional policy. Also, if the sum of processing times is the same, the average cycle time is also the same in the proposed policy. We show this with figure for $p_{1}=1$ and $p_{2}=2$. The variation of workers' processing stations according to the time is shown in Figure 6. In Figure 6, worker 1 walks back to the first station at time 4, and passes worker 2 at times 2, 6, and 10 . Using both policies, the faster worker 1 processes at many stations; therefore, the cycle time decreases. On the other hand, in the case in which $p_{1}=2$ and $p_{2}=3$, the cycle time of the proposed policy is the same as the traditional rule. This is because even if both policies are adapted, one worker has to wait for another worker, de- 
pending on processing time. This proposed policy is more effective, especially if the processing time of one worker is a multiple of the processing time of another worker.

\subsection{Effect of the Real Value Case}

In the previous three subsections, the processing time is assumed to be an integer. In this subsection, we analyze the case in which one worker's processing time is one, but another's is a real number. The result is shown in Table 4. These results show that the cycle time decreases as ratio of processing time. For example, the processing time when $p_{1}=1$ and $p_{2}=1.5$ is half that of the case when $p_{1}=2$ and $p_{2}=3$. Therefore, the cycle time decreases from 5 to 2.5 . However, when worker processing times are not integral multiplies of any other worker's processing time, blocking occurs frequently. Therefore, the cycle time increases more than the ratio of processing time.

Table 4. Result for real value case.

\begin{tabular}{|c|c|c|c|}
\hline \multirow{2}{*}{$p_{1}$} & \multirow{2}{*}{$p_{2}$} & \multicolumn{2}{|c|}{ Average cycle time } \\
\cline { 3 - 4 } & & Proposed & Traditional \\
\hline \hline 1 & 1.5 & 2.5 & 2.5 \\
\hline 1.5 & 1 & 2.5 & 3 \\
\hline
\end{tabular}

\subsection{Extending to More Than Three Workers}

In this section, based on the results of the previous four sections, we try to give insight to extend our work to more than three workers. Results show that when slower workers process less, a short cycle time can be achieved. This is because blocking by slower workers seldom occurs. Therefore, we should set policies in which slower workers process less. To do so, the proposed policy is preferable even if there are more than three workers.

\section{CONCLUSION}

This research focuses on the self-balancing production line with stations. First, we propose a new policy with two measures: allowing passing and walking back. Based on these two measures, we analyze the effect of each and compare to the rule in the traditional selfbalancing production line. Our results show that, using only one of these policies does not achieve a good result compared to the traditional rule. However, by integrat- ing these policies, we can derive smaller cycle times than that of the traditional policy. Moreover, based on the results, we give aninsight to extend this to more than three workers.

In our research, we only give insight to extend more than three workers. Analyzing more than three workers is future research work.

\section{REFERENCES}

Armbruster, D. and Gel, E. S. (2006), Bucket brigades revisited: are they always effective?, Euro. J. Operational Research, 172, 213-229.

Bartholdi, J. J. and Bunimovich L. A. (1999), Dynamics of two- and three-worker 'Bucket Brigade' production lines, Oper. Res., 47, 488-491.

Bartholdi, J. J. and Eisenstein, D. D. (1996), A production line that balances itself, Oper. Res., 44, 21-34.

Bartholdi, J. J., Eisenstein, D. D., and Foley, D. D. (2001), Performance of bucket brigades when work is stochastic, Oper. Res., 49, 488-491.

Buzacott, J. A. (2002), The impact of worker difference on production system output, Int. J. Production Economics, 78, 37-44.

Hirotani, D., Morikawa, K., and Takahashi, K. (2009), Analysis of self-balancing production line with stations, non preemption and constant working speed, Proceeding of the 9th Asia-Pacific Industrial Engineering and Management System Conference, Kitakyushu, Japan, 1986-1996.

Hirotani, D., Morikawa, K., and Takahashi, K. (2008), Analysis and design of self-balancing production line with large number of stations and workers, Proceeding of the 8th Asia-Pacific Industrial Engineering and Management System Conference, Bali, Indonesia, 936-943.

Hirotani, D., Myreshka, Morikawa, K., Takahashi, K. (2006), Analysis and design of the self-balancing production line, Computers and Industrial Engineering, 50, 488-502.

McClain, J. O., Schultz K. L., and Thomas L. J. (2000), Management of worksharing systems, Manufacturing and service operations management, 2, 49-67.

Nakade, K., Ohno, K. (2003), Separate and carousel type allocations of workers in a U-shaped production line, Euro. J. Operational Research, 145, 403424.

Scholl, A. (1995), Balancing and Sequencing of Assembly Lines, Physica-Verlag, Heidelberg, NY. 